\title{
Differential subcellular localization of functionally divergent survivin splice variants
}

\author{
C Mahotka', J Liebmann ${ }^{1}$, M Wenzel $^{1}$, CV Suschek ${ }^{2}$, \\ M Schmitt ${ }^{3}$, HE Gabbert ${ }^{1}$ and CD Gerharz ${ }^{*, 1}$ \\ ${ }^{1}$ Institute of Pathology, Heinrich Heine-University, Duesseldorf, Germany \\ 2 Institute of Immunobiology, Heinrich Heine-University, Duesseldorf, Germany \\ ${ }^{3}$ Department of Gastroenterology, Hepatology and Infectiology, Heinrich Heine- \\ University, Duesseldorf, Germany \\ * Corresponding author: Claus Dieter Gerharz, Institute of Pathology, Heinrich \\ Heine-University, Moorenstr. 5, D - 40225 Duesseldorf, Germany. \\ Tel: 49-211-811 8348; Fax: 49-211-811 8353; \\ E-mail: gerharz@med.uni-duesseldorf.de
}

Received 15.1.02; revised 13.6.02; accepted 17.6.02

Edited by D Altieri

\begin{abstract}
Survivin is an inhibitor of apoptosis protein (IAP) that is markedly overexpressed in most cancers. We identified two novel functionally divergent splice variants, i.e. non-antiapoptotic survivin-2B and antiapoptotic survivin- $\Delta E \times 3$. Because survivin-2B might be a naturally occurring antagonist of antiapoptotic survivin variants, we analyzed the subcellular distribution of these proteins. PSORT II analysis predicted a preferential cytoplasmic localization of survivin and survivin-2B, but a preferential nuclear localization of survivin- $\Delta$ Ex3. GFP-tagged survivin variants confirmed the predicted subcellular localization and additionally revealed a cell cycle-dependent nuclear accumulation of survivin- $\Delta E \times 3$. Moreover, a bipartite nuclear localization signal found exclusively in survivin- $\Delta$ Ex 3 may support cytoplasmic clearance of survivin- $\Delta E \times 3$. In contrast to the known association between survivin and microtubules or centromeres during mitosis, no corresponding co-localization became evident for survivin- $\Delta \mathrm{Ex} 3$ or survivin-2B. In conclusion, our study provided data on a differential subcellular localization of functionally divergent survivin variants, suggesting that survivin isoforms may perform different functions in distinct subcellular compartments and distinct phases of the cell cycle.

Cell Death and Differentiation (2002) 9, 1334-1342. doi:10.1038/ sj.cdd. 4401091
\end{abstract}

Keywords: survivin; apoptosis; alternative splicing; cancer

Abbreviations: IAP, inhibitor of apoptosis; BIR, baculovirus inhibitory repeat; GFP, green fluorescent protein; PSORT, program for detecting the protein sorting signals and predicting their subcellular localization; NLS, nuclear localization signal

\section{Introduction}

Deregulation of apoptosis is involved in carcinogenesis by abnormally prolonging cell survival, facilitating the accumulation of transforming mutations and promoting resistance to immunosurveillance. ${ }^{1}$ The molecular pathways in the execution of apoptotic cell death are highly conserved evolutionarily, as are their regulators. ${ }^{2,3}$ A novel family of proteins that negatively regulates cell death is the inhibitor of apoptosis (IAP) protein family. Originally identified in the baculoviral genome, ${ }^{4}$ cellular homologues of IAPs have also been identified in yeast, nematode, drosophila and vertebrates. ${ }^{5-7}$ IAPs are characterized by one or multiple motifs called baculoviral IAP repeat (BIR), which is considered to be the main mediator of antiapoptotic function, enabling IAPs to inhibit the action of cystein proteases (caspases). ${ }^{8,9}$

Survivin was identified as a novel human member of the IAP family that contains only a single BIR domain. ${ }^{10}$ It was shown that survivin inhibits processing of procaspase-3 and -7 and specifically binds both active caspases in a cell free system, ${ }^{11,12}$ although these actions are still a matter of controversial debate. ${ }^{12-14}$ Moreover, survivin was shown to inhibit caspase- 9 actions. ${ }^{15}$ Survivin expression exhibited a marked cell cycle periodicity with a pronounced upregulation during the G2/M phase followed by a rapid decline in the G1 phase. ${ }^{16}$ The degradation of survivin protein by the ubiquitin-proteasome pathway was found to be strictly regulated by an intact carboxyterminal region downstream of the BIR domain. ${ }^{17}$ In fact, survivin may act at the interface between apoptosis and cell cycle regulation, as evident from recent observations demonstrating complex formation between survivin and centromeres ${ }^{18-20}$ or the cyclin dependent kinase $\mathrm{Cdk} 4^{21,22}$ as well as survivin phosphorylation by the $\mathrm{p} 34^{\mathrm{cdc} 2}-$ cyclinB1 $^{\text {complex. }}{ }^{15}$

The intracellular topography of survivin was described as predominantly cytosolic with a ratio of cytosolic vs nuclear survivin of $6: 1,{ }^{23}$ taking into account the breakdown of the nuclear membrane in mitotic cells. On the subcellular level, it was shown that survivin binds to microtubules of the mitotic spindle and the midzone, ${ }^{16}$ to centrosomes ${ }^{18,20}$ or to kinetochores. ${ }^{19}$ The reported diversity of subcellular localization could result from the analysis of different survivin fusion proteins or from the use of diverse survivin-specific antibodies recognizing distinct epitopes, ${ }^{20,23}$ but also from differences in $\mathrm{Thr}^{34}$ phosphorylation ${ }^{15,23}$ or cell culture conditions (e.g. treatment with paclitaxel). ${ }^{24}$

Survivin is expressed in many fetal and some proliferating adult tissues, ${ }^{25,26}$ whereas no transcripts were detected in a variety of resting adult tissues. ${ }^{10,26}$ Most strikingly, survivin was found in the most common human cancers, suggesting that cancer cells return to a fetal pattern of survivin expression. Re-expression of survivin may enhance cell viability and enable the neoplastic cell to overcome the cytotoxic effects of chemotherapeutic agents. 
In fact, initial studies in neuroblastomas, colorectal carcinomas, non-small-cell lung cancer, and bladder cancer indicated a correlation between the re-expression of survivin and an unfavorable course of the disease, proposing survivin expression as a potential prognostic factor. ${ }^{27,28}$

Recently, we identified two functionally divergent splice variants of survivin, which are characterized by a common structural alteration of their BIR domain: ${ }^{29}$ the loss of exon 3 in survivin- $\Delta \mathrm{Ex} 3$ results in a BIR domain truncated at amino acid position 74 and a frame shift of the carboxyterminus. Despite these profound structural alterations, the antiapoptotic potential of survivin- $\Delta \mathrm{Ex} 3$ was largely preserved. Remarkably, the survivin- $\Delta$ Ex3-carboxyterminus is completely different from the survivin carboxyterminus, which might interfere with degradation of survivin$\Delta E \times 3$ protein by ubiquitin tagging. Survivin- $\Delta E \times 3$, therefore, could evade the cell cycle-specific degradation by the ubiquitin-proteasome pathway, known for survivin. In survivin-2B, inclusion of the cryptic exon $2 \mathrm{~B}$ encodes the insertion of 23 additional amino acids into the BIR domain at essentially the same position, where the BIR domain of survivin- $\Delta E \times 3$ is truncated. Because survivin-2B exhibited a loss of antiapoptotic potential, ${ }^{29}$ we hypothesized that this survivin variant might be a naturally occurring antagonist of antiapoptotic survivin and survivin- $\Delta \mathrm{Ex} 3$, possibly by competitive binding to common interaction partners, as reported for survivin and survivin-2B interactions with polymerized tubulin. ${ }^{30}$

Because competitive inhibition would require identical subcellular localization for physical interaction, we comparatively analyzed the subcellular distribution of all survivin variants in interphase cells and during mitosiswith a special focus on co-localization with the kinetochorebinding passenger protein CENP-F and midbodies.

\section{Results}

\section{PSORT II analysis predicts different subcellular localization of antiapoptotic and non-antiapoptotic survivin variants}

Using the PSORT II program which applies algorithms for protein sorting signals we analyzed the potential subcellular localization of the different survivin variants. As shown in Table 1, a largely congruent subcellular localization was

Table 1 Prediction of subcellular localization by PSORT II analysis

\begin{tabular}{lrcc}
\hline Localization & $\begin{array}{c}\text { Survivin } \\
{[\%]}\end{array}$ & $\begin{array}{c}\text { Survivin- } \Delta \text { Ex3 } \\
{[\%]}\end{array}$ & $\begin{array}{c}\text { Survivin-2B } \\
{[\%]}\end{array}$ \\
\hline Cytoplasm & 56.5 & 26.1 & 52.2 \\
Nucleus & 17.4 & 52.2 & 21.7 \\
Cytoskeleton & 0.0 & 8.7 & 0.0 \\
Golgi apparatus & 0.0 & 4.3 & 0.0 \\
Plasma membrane & 4.3 & 4.3 & 4.3 \\
ER & 4.3 & 0.0 & 4.3 \\
Peroxysomes & 0.0 & 4.3 & 0.0 \\
Mitochondria & 13.0 & 0.0 & 13.0 \\
Lysosomes & 4.3 & 0.0 & 4.3 \\
\hline
\end{tabular}

predicted for survivin and survivin-2B, the cytoplasm being the most probable localization of both variants. Of note, the predicted subcellular localization of survivin matches the differential distribution of survivin reported experimentally. ${ }^{23}$ In contrast, survivin- $\Delta \mathrm{Ex} 3$ was predicted to exhibit an inverse distribution pattern, the nucleus being the most probable localization of this survivin variant.

\section{Different subcellular localization of antiapoptotic and non-antiapoptotic survivin variants}

Because no antibodies are currently available to differentiate the subcellular localization of endogenous survivin- $\Delta \mathrm{Ex} 3$ and survivin-2B from survivin, we transfected HepG2 cells with pEGFP vector constructs containing the different survivin variants. In these transfectants, the survivin variants are constitutively expressed under the control of the CMV promoter, which is not regulated by the cell cycle. In consequence, mRNA stability and the capacity of survivindegrading systems will determine the extent of protein accumulation, but not its subcellular localization. The functionality of the respective survivin-GFP fusion proteins was tested by a CD95-specific apoptosis assay, since HepG2 cells are known to be sensitive for CD95-mediated apoptosis. Using the agonistic $\mathrm{CH} 11$ antibody for CD95 activation, survivin-GFP and survivin- $\triangle$ Ex3-GFP exhibited preserved antiapoptotic activity, whereas survivin-2B-GFP revealed a loss of antiapoptotic potential (Figure 1), as previously observed for untagged survivin variants as well. ${ }^{29}$

As predicted by PSORT II analysis, confocal laser scanning microscopy confirmed the cytoplasmic colocalization of survivin and survivin-2B, whereas survivin- $\Delta \mathrm{Ex} 3$ exhibited an exclusively nuclear accumulation (Figure 2A).

Additional immunostaining for the $\mathrm{Ki}-67$ protein, which is present during all active phases of the cell cycle (late G1, S, G2, M) and absent from resting G0 cells, ${ }^{31}$ revealed cytoplasmic accumulation of survivin and survivin-2B in both Ki-67-negative and Ki-67-positive cells (Figure 2A). In contrast, nuclear accumulation of survivin- $\Delta \mathrm{E} \times 3$ was exclusively found in Ki-67-positive cells (Figure 2A). Because the CMV promoter of our vector constructs is not regulated by the cell cycle, survivin- $\Delta \mathrm{Ex} 3$ should be constitutively transcribed and translated during all phases of the cell cycle. Therefore, the selective accumulation of survivin- $\Delta \mathrm{Ex} 3$ in $\mathrm{Ki}-67$-positive nuclei may suggest a cell cycle-dependent mechanism of survivin- $\Delta \mathrm{Ex} 3$ translation in Ki-67-positive or protein degradation in Ki-67-negative cells. First hints for a role of survivin- $\Delta$ Ex3 degradation were obtained from a cycloheximide block experiment (Figure 2B) demonstrating a more rapid turnover of ectopic survivin- $\Delta \mathrm{Ex} 3$ when compared to the protracted degradation of ectopic survivin. This mechanism could also be relevant for the endogenous survivin- $\Delta \mathrm{E} \times 3$, sustaining elevated levels in the active (Ki-67-positive) phases of the cell cycle (i.e. late $\mathrm{G} 1, \mathrm{~S}, \mathrm{G} 2$, and $\mathrm{M}$ ), parallel to the cell cycle-dependent upregulation of endogenous survivin. However, degradation of survivin- $\Delta \mathrm{E} \times 3$ with its markedly modified carboxyterminus presumably is different from the ubiquitin-proteasome pathway, which depends on an intact survivin carboxyterminus. ${ }^{17}$ Nevertheless, this as yet 


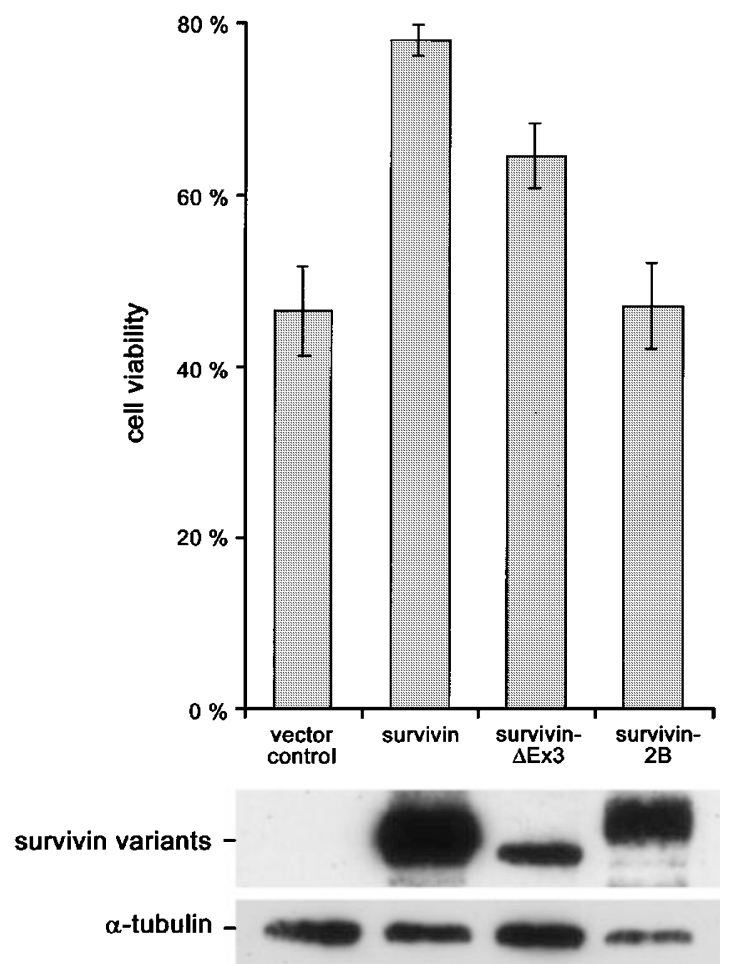

Figure 1 Differential antiapoptotic potential of survivin splice variants in the human hepatoma cell line HepG2. (a) Cell death was induced by the CD95specific agonistic $\mathrm{CH} 11$-antibody. Survivin and survivin- $\triangle \mathrm{Ex} 3$ inhibit CD95specific apoptosis, whereas survivin-2B has completely lost its antiapoptotic potential. The percentage of transfected cells was indirectly determined by microscopic evaluation of $\beta$-galactosidase expressing cells. The transfection efficiency was about $40 \%$ in all transfection experiments. Data are means of three independent experiments. (b) Immunoblot analysis of these transfectants with anti-survivin antibody revealed stable and comparable levels of expression for the indicated survivin variants. Of note, less intensive survivin$\Delta$ Ex3 signals may be due to the modified carboxyterminus, resulting in incomplete epitope covering by polyclonal antibodies originally raised against full-length survivin

hypothetic alternative pathway could be even more efficient, as suggested in our experiments by the lack of ectopic survivin- $\Delta \mathrm{Ex} 3$ in Ki-67-negative cells in contrast to cytoplasmic accumulation of ectopic survivin and survivin$2 \mathrm{~B}$.

Because survivin may act, inter alia as a chromosomal passenger protein that is closely associated with centromeres during mitosis, ${ }^{19,20}$ we further compared the subcellular localization of the different survivin variants using CENP-F as a centromere marker (Figure $3 A, B, C$ ). CENP-F is a component of the nuclear matrix that gradually accumulates during the cell cycle and reaches peak levels in G2/M phase cells. CENP-F assembles onto the centromeres during mitosis. ${ }^{32}$

In accordance with previous observations, ${ }^{19,20}$ we observed a specific translocation of survivin to the centromeric regions during mitosis, as evident from the close co-localization of signals for survivin and CENP-F (Figure $3 \mathrm{~A} / \mathrm{b}$ ). In contrast, the loss of the nuclear membrane during mitosis was accompanied by a redistribution of survivin- $\Delta \mathrm{Ex} 3$ and survivin-2B throughout the cell. Even

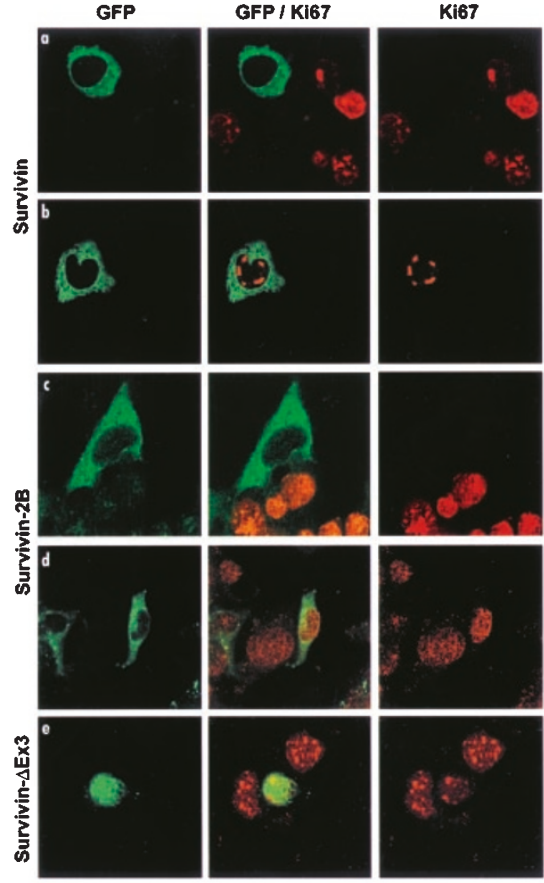

B

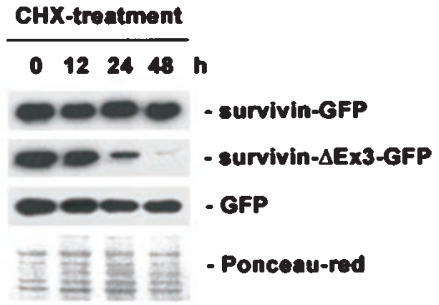

Figure 2 (A), Differential localization of survivin variants. Immunofluorescence staining for $\mathrm{Ki}-67$ (red) and direct fluorescence emission by GFP-tagged survivin/survivin- $\Delta \mathrm{E} \times 3 /$ survivin-2B (green) in HepG2 cells grown on coverslips. Left panel shows survivin-GFP fusion proteins. Survivin and survivin-2B are located in the cytoplasm, whereas survivin- $\Delta \mathrm{Ex} 3$ is located in the nucleus. Right panel shows nuclear staining with the cell proliferation marker Ki-67, which specifically stains cells in all active phases of the cell cycle (late G1, S, $\mathrm{G} 2, \mathrm{M})$. The middle panel shows the overlay of $\mathrm{Ki}-67$ and survivin localization. Recombinant survivin and survivin-2B were expressed in Ki-67-negative $(\mathbf{a}, \mathbf{c})$ and Ki-67-positive (b, d) cells, whereas survivin- $\Delta$ Ex3 could be detected only in Ki-67-positive cells (e). (B) Comparison of survivin vs survivin- $\Delta \mathrm{Ex} 3$ protein stability. After translation inhibition with cycloheximide, survivin- $\triangle$ Ex3-GFP degrades faster than survivin-GFP or GFP from vector control. Experiments were repeated four times with highly comparable results. GFP (present as a tag in all survivin variants) was used as additional control of protein stability after $\mathrm{CHX}$ treatment. Comparable protein loading was shown after Ponceaured staining of the blotting membrane

more striking was the observation that neither survivin$\Delta \mathrm{Ex} 3$ nor survivin-2B showed the close association with the centromere marker CENP-F, observed for survivin. Thus, co-localizing signals with CENP-F were detected extremely seldom only for survivin- $\Delta E x 3$ (Figure $3 B / b$ ) and not at all for survivin-2B (Figure $3 \mathrm{C} / \mathrm{b}$ ). (Interestingly, the formation of dual foci characteristic for CENP-F immunostaining of centromeric regions seems to be disturbed in survivin$\Delta$ Ex3 transfectants as well). Moreover, only survivin, but not survivin- $\Delta \mathrm{Ex} 3$ or survivin-2B) was detectable in the 

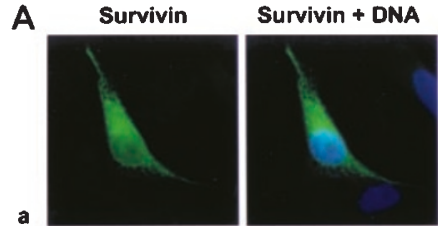

CENP.F
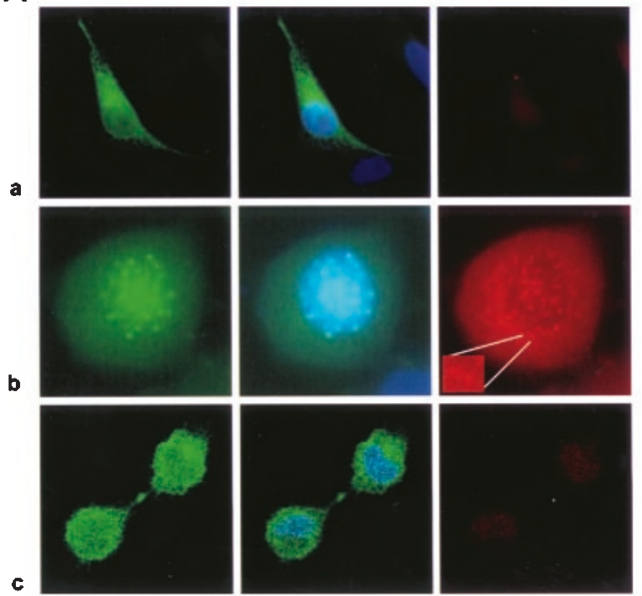

B
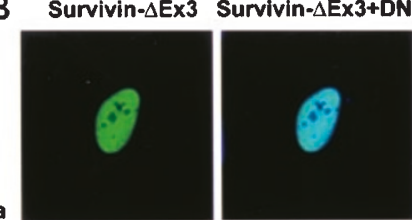

CENP.F
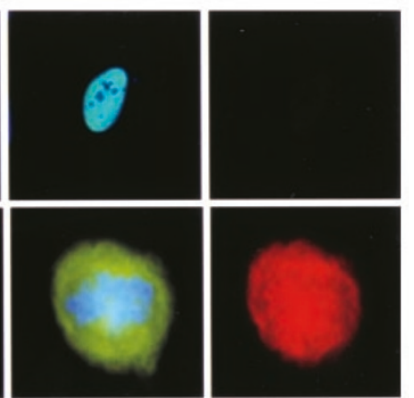

b
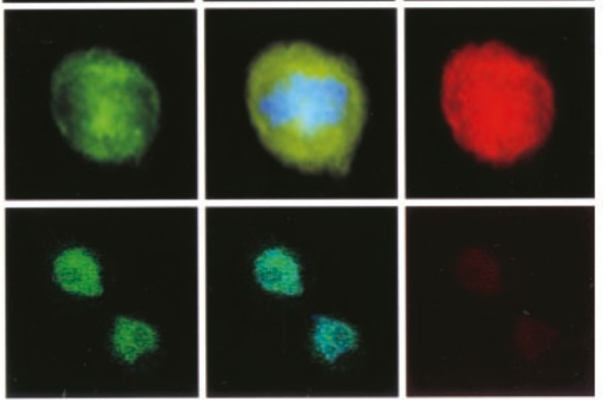

c
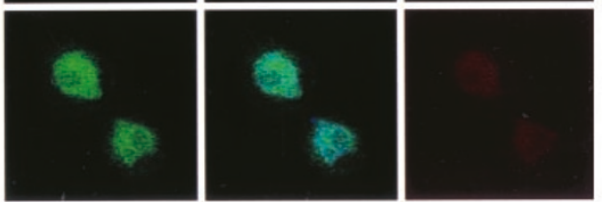

C Survivin-2B
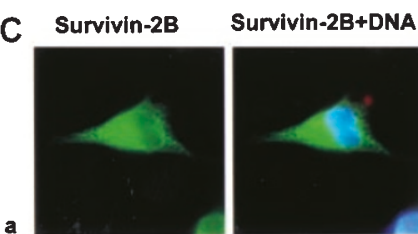

CENP.F
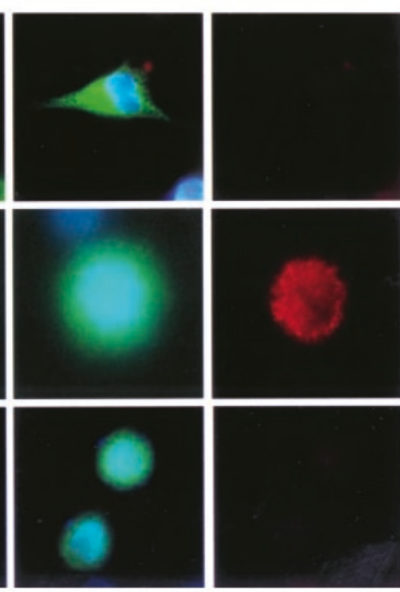

Figure 3 (A), Subcellular localization of survivin-GFP during the cell-cycle. The left panel shows survivin-GFP expression, the middle panel survivin-GFP and DNA staining (DAPI). The right panel shows a CENP-F staining. Of note, CENP-F gradually accumulates during the cell cycle with peak levels in G2 and $\mathrm{M}$ phase cells and rapid degradation upon completion of mitosis. ${ }^{32}$ Paired foci of CENP-F immunostaining clearly mark the centromeres (b) by binding to the outer plates of the kinetochores. Survivin-GFP is predominantly localized to the cytoplasm in interphase (a), accumulates in distinct foci on centromeres in metaphase (b), and is found in the midbody during cytokinesis (c). (B), Subcellular localization of survivin- $\Delta$ Ex3-GFP during the cell cycle. The left midbodies of telophase (Figure $3 \mathrm{~A} / \mathrm{c}, \mathrm{B} / \mathrm{c}$ and $\mathrm{C} / \mathrm{c}$ ), suggesting a disturbed interaction with the microtubules of the midbodies as well.

\section{A bipartite nuclear targeting sequence in survivin- $\Delta \mathrm{Ex} 3$ and its role in differential subcellular localization}

Because the nuclear accumulation of survivin- $\Delta E x 3$ may be due to a specific nuclear localization signal (NLS), absent in both survivin and survivin-2B, we used PSORT II analysis for the identification of three different NLS recognition patterns: Whereas no 'pat4' NLS pattern could be detected in any survivin variant, the 'pat7' recognition pattern identified the NLS sequence PTIRRKN ${ }^{84}$ in survivin- $\Delta E x 3$, but not in the other survivin variants. Interestingly, this sequence was also found to be a part of the bipartite NLS RKPTIRRKNLRKLRRKC ${ }^{92}$, identified by PSORT II analysis exclusively in survivin- $\Delta E \times 3$. This bipartite NLS is generated in survivin- $\Delta \mathrm{Ex} 3$ by alternative splicing and the frame shift of exon 4 (Figure 4), whereas no fragment of this bipartite NLS motif was present in survivin and survivin-2B. The bipartite NLS sequence of survivin- $\Delta \mathrm{Ex} 3$ encompasses a spacer of 11 amino acids and fully meets the consensus sequence criteria defined for other bipartite nuclear

survivin- $\Delta \mathrm{E} \times 3$
glucocorticoid receptor
progesteron receptor
androgen receptor
estrogen receptor
erb-A
thyroid $\beta$ receptor
HSF2-1
HSF2- 2
SRY
PARP

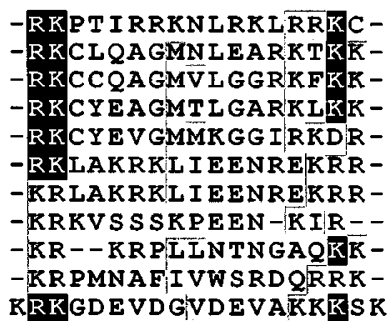

Figure 4 Alignment of bipartite nuclear localization signals of survivin- $\Delta \mathrm{E} \times 3$ and other proteins. The bipartite NLS of survivin- $\Delta E \times 3$ has marked homology to steroid receptor NLSs. Interestingly, the bipartite NLS of survivin- $\Delta \mathrm{Ex} 3$ also encompasses basic amino acids of the 'pat7'-NLS PTIRRKN. Alignment of selected bipartite nuclear localization signals was made by ClustalW and enhanced with Boxshade. Darker shading is of residues that are highly conserved; lighter shading is of less well conserved residues, and residues that are not shaded are not conserved

panel shows survivin- $\Delta$ Ex3-GFP expression, the middle panel survivin- $\Delta$ Ex3 GFP and DNA staining (DAPI), the right panel shows staining for CENP-F. Survivin- $\triangle$ Ex3-GFP is exclusively localized to the nucleus in interphase, with respect to Ki-67 staining (cf. Figure 2) presumably in late $\mathrm{G} 1, \mathrm{~S}$ and $\mathrm{G} 2$ periods of the interphase (a). In metaphase cells (b) survivin- $\triangle$ Ex3-GFP is distributed throughout the cell, indicating a loss of nuclear compartmentalization after dissolution of the nuclear membrane. In contrast to the close co-localization of survivin-GFP and CENP-F (cf. A/b), co-localizing signals for survivin- $\Delta$ Ex3GFP and CENP-F were only rarely observed in a very small number of cells. Of note, the formation of dual foci characteristic for CENP-F immunostaining of centromeric regions (cf. $\mathbf{A} / \mathbf{b}$ ) seems to be disturbed in survivin- $\Delta \mathrm{Ex} 3$ transfectants. During cytokinesis (c) no survivin- $\triangle$ Ex3-GFP is observed at the midbody. (C), Subcellular localization of survivin-2B-GFP during the cell cycle. The left panel shows survivin-2B-GFP expression, the middle panel survivin-2B-GFP and DNA staining (DAPI), the right panel shows staining for CENP-F. Survivin-2B-GFP is localized in the cytoplasm in interphase (a). In metaphase cells (b) survivin-2B-GFP shows no centromeric association. At telophase (c) no survivin-2B-GFP is found at the midbody 
targeting motifs (consensus sequence according to Jans and Hübner: ${ }^{33}(\mathrm{~K} / \mathrm{R})(\mathrm{K} / \mathrm{R})-(10-12$ amino acid spacer $)-(\mathrm{K} /$ $R)(K / R)(K / R))$. The differential subcellular localization of the survivin variants was further suggested by an NLS score of +0.36 for survivin- $\Delta$ Ex3 vs -0.47 each for survivin and survivin-2B. Interestingly, a closely corresponding bipartite NLS could be found in survivin- $\Delta$ Ex118 (Figure 5 ), the recently identified porcine homologue of survivin- $\Delta \mathrm{Ex} 3{ }^{7}$

To further evaluate the relevance of the bipartite NLS within survivin- $\Delta \mathrm{Ex} 3$, an NLS-deleted mutant was generated. Interestingly, however, the nuclear localization of the NLS-deleted survivin- $\triangle$ Ex3-GFP variant was preserved, but now accompanied by an additional cytoplasmic

BIR

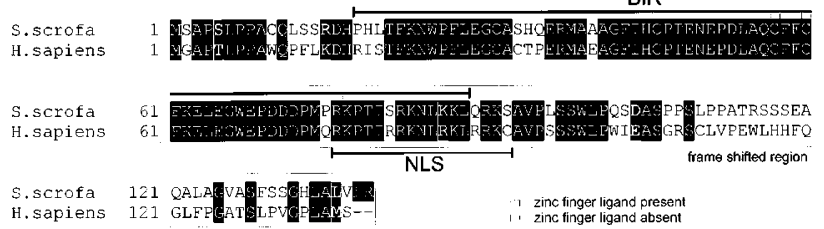

Figure 5 Alignment, using ClustalW and enhanced with Boxshade, of complete amino acid sequences of survivin- $\Delta \mathrm{Ex} 3$ and the survivin-homologue $\Delta 118$ from $S$. scrofa. The first part of the carboxyterminus including the NLS of survivin- $\Delta \mathrm{Ex} 3$ is conserved in survivin- $\Delta 118$. Both alternative splice variants have a high identity, which could indicate similar function in both species. Of note, the second part of the carboxyterminus of survivin- $\Delta 118$ from $S$. scrofa shows only weak homology and has - in contrast to survivin- $\Delta \mathrm{Ex} 3-\mathrm{a}$ higher probability for a coiled coil (data not shown). Darker shading is of residues that are highly conserved; lighter shading is of less well conserved residues, and residues that are not shaded are not conserved. The frame shifted region of the carboxyterminus is boxed distribution (Figure 6). Corresponding observations have previously been reported after deletion of the essential NLS involved in nuclear sorting of human uracil-DNA glycosylase. ${ }^{34}$ The bipartite NLS, therefore, may not directly mediate the selective accumulation of survivin$\Delta \mathrm{Ex3}$ in the nuclear compartment, but, nevertheless, seems to be functionally active in that it supports survivin- $\Delta$-Ex3 clearance out of the cytoplasmic compartment. In consequence, additional NLS-flanking sequences (including non-basic residues) in the strongly modified carboxyterminus are necessary for the exclusive nuclear accumulation of survivin- $\Delta \mathrm{Ex} 3$.

\section{Discussion}

As recently demonstrated by our group ${ }^{7,29}$ and subsequently confirmed by others, ${ }^{30,35}$ alternative splicing may be important for the fine tuning of survivin actions. In close analogy to other regulators of apoptosis, e.g. $\mathrm{Bcl}-\mathrm{X}_{\mathrm{L}}$ or $\mathrm{Bcl}-\mathrm{X}_{\mathrm{S}}$, the actions of antiapoptotic survivin and survivin- $\Delta \mathrm{Ex} 3$ might be counterbalanced by the non-antiapoptotic survivin-2B variant. Functional antagonism between antiapoptotic and nonantiapoptotic survivin variants could result from competitive binding of heterologous interaction partners or-with respect to the recently observed dimer formation of survivin ${ }^{13,36,37}$ from the formation of inactive survivin: survivin-2B heterodimers. In fact, our studies revealed an important prerequisite for physical interaction, i.e. a cytoplasmic co-localization of survivin and survivin-2B. In contrast, survivin- $\Delta \mathrm{Ex} 3$ exhibited a preferential localization in the nuclear compartment from late $\mathrm{G} 1$ to $\mathrm{G} 2$ phase.

\section{Survivin- $\Delta E \times 3^{-N L S}$-GFP}

a
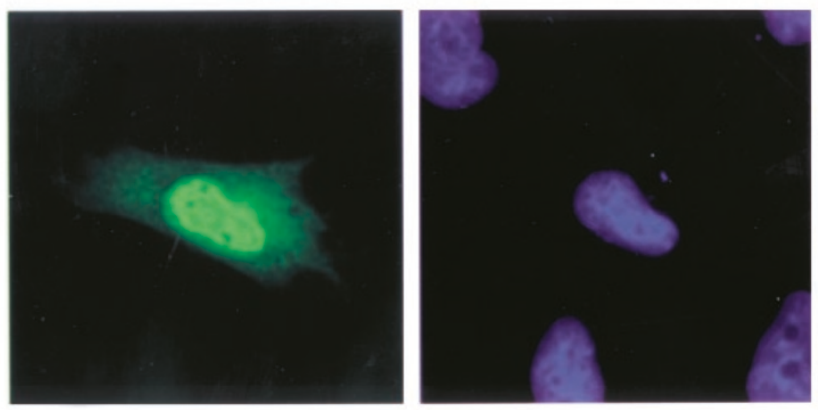

b

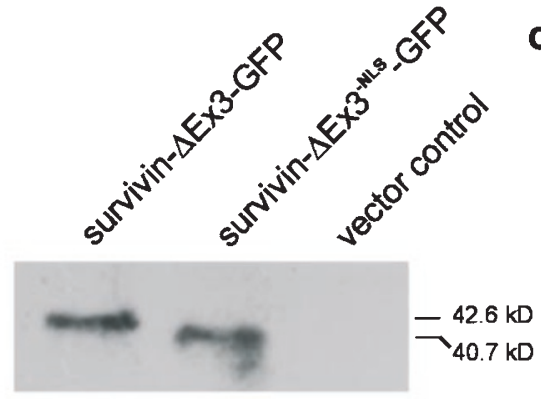

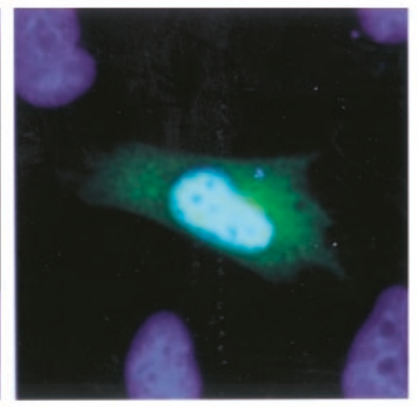

DNA

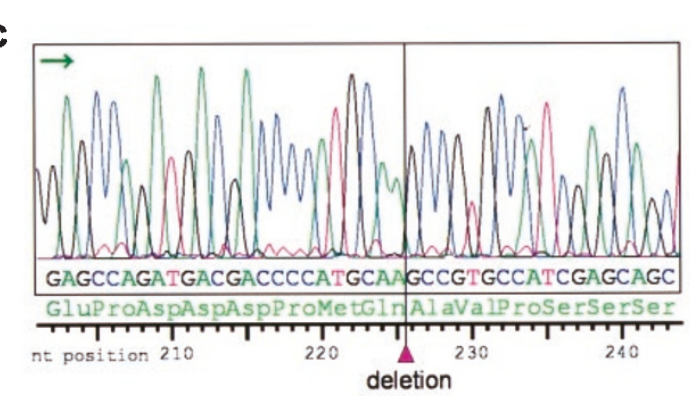

Figure 6 Deletion of the bipartite NLS in survivin- $\Delta$ Ex3 $3^{-N L S}$-GFP results in both cytoplasmic and nuclear localization, suggesting that the bipartite NLS does not directly modulate nuclear accumulation, but rather supports clearance out of the cytoplasmic compartment (a). The expression of survivin- $\Delta$ Ex $3^{-N L S}$-GFP constructs was checked by immunoblotting using anti-survivin antibodies (b) and the accuracy of deletion was proven by DNA sequencing (c) 
Because survivin may act, inter alia as a centromerebinding chromosomal passenger protein associated with INCENP and Aurora-B kinase, ${ }^{18-20}$ we additionally compared the subcellular localization of survivin, survivin- $\triangle \mathrm{E} \times 3$ and survivin-2B during mitosis, using CENP-F as a centromere marker protein. CENP-F is known to bind specifically at the outer plate of the kinetochores as a chromosomal passenger protein, producing characteristic dual foci in CENP-F immunostaining. In accordance with previous reports, survivin showed a close colocalization with the centromeres in our experiments as well. Survivin- $\Delta \mathrm{Ex} 3$ and survivin-2B, however, did not exhibit such a close association to the centromere region. Thus, sparse co-localizing signals for survivin- $\Delta \mathrm{E} \times 3$ and CENP-F were observed in a very few cells only. In contrast to survivin, the loss of the nuclear membrane during mitosis was accompanied by a redistribution of survivin-Ex3 throughout the cell and no accumulation in the midbodies became evident during cytokinesis. With respect to the nuclear accumulation of survivin- $\Delta \mathrm{Ex} 3$ during late $\mathrm{G} 1, \mathrm{~S}$ and $\mathrm{G} 2$, as indicated by Ki-67 counterstaining, it is tempting to speculate that survivin$\Delta$ Ex3 might also play a regulatory role for cell cycle progression at the mitotic spindle checkpoint as previously demonstrated for survivin. ${ }^{16}$ On the other hand, survivin- $\Delta \mathrm{Ex} 3$ was not found in midbodies at late telophase, suggesting that survivin- $\Delta \mathrm{E} \times 3$ in contrast to survivin, fails to interact with microtubules at the mitotic spindle midzone. Although survivin-2B has been suggested to be a microtubule binding protein in vitro, ${ }^{30}$ we did not observe survivin-2B binding at midbodies either. The actual molecular interaction partners for survivin$\Delta \mathrm{Ex} 3$ and survivin-2B, however, still remain elusive. Thus, it is still unclear, whether survivin- $\Delta$ Ex3 may interact, at least to some degree, with centromeres or whether we observed an incidental co-localization without functional relevance. The latter view is supported by recent reports indicating that structural relatives of survivin- $\Delta E \times 3$ and survivin-2B, i.e. survivin mutants with a truncated BIR domain or a deletion of the carboxyterminus, also fail to localize correctly to the centromeres or midbodies. ${ }^{19}$

Expression of endogenous survivin has previously been found to be regulated at the transcriptional and the posttranscriptional level. Thus, the combined action of $\mathrm{G} 1$ transcriptional repressor elements within the survivin promoter $^{38}$ and cell cycle-dependent degradation via the ubiquitin-proteasome pathway ${ }^{17}$ results in upregulation of endogenous survivin at $\mathrm{G} 2 / \mathrm{M}$ and downregulation at G1. Moreover, endogenous survivin is rapidly turned over with a half-life of about 30 min. ${ }^{17}$ Little, however, is known so far about the mechanisms determining the protein levels of endogenous survivin- $\Delta E \times 3$ and survivin-2B, although the mRNA levels of both variants have been found to be constantly exceeded by survivin mRNA levels in different tumor types. ${ }^{29,39,40}$ Because G1 transcriptional repressor elements within the survivin promoter are relevant for the transcription of all survivin variants, a cell cycle-dependent transcription of survivin- $\Delta \mathrm{Ex} 3$ and survivin-2B seems to be feasible as well. The actual protein levels of endogenous survivin- $\Delta \mathrm{E} \times 3$ and survivin-2B, however, will depend on both the splicing machinery regulating the relative proportions of each mRNA variant and the stability of each mRNA or protein variant. Interestingly in this context, not all survivin variants seem to be substrates for the ubiquitinproteasome pathway of degradation, which becomes effective in the cytoplasm only and requires an intact carboxyterminus. ${ }^{17}$ Therefore, both the nuclear translocation and the strongly modified carboxyterminal region may effectively protect survivin- $\Delta \mathrm{Ex} 3$ from rapid degradation by the ubiquitin-proteasome pathway. Nevertheless, our cycloheximide block experiments suggested the existence of an alternative pathway of survivin- $\Delta \mathrm{Ex} 3$ degradation, which resulted in an accelerated turnover of ectopic survivin- $\Delta \mathrm{Ex} 3$ when compared to ectopic survivin. Further experiments will have to show to what extent this alternative pathway may contribute to the cell cycle-dependent nuclear accumulation of survivin- $\Delta \mathrm{Ex} 3$.

PSORT II analysis suggested that nuclear accumulation of survivin- $\Delta \mathrm{E} \times 3$ could also be related to a novel bipartite nuclear localization signal, which was not detected in survivin and survivin-2B. Our transfection experiments with an NLS-deleted survivin- $\Delta \mathrm{Ex} 3$ mutant, however, demonstrated that the bipartite NLS does not directly mediate the selective nuclear accumulation, but rather supports its clearance out of the cytoplasmic compartment. Further studies, therefore, will have to elucidate the molecular mechanisms resulting in the selective nuclear accumulation of survivin- $\Delta \mathrm{Ex} 3$ in Ki-67-positive cells.

Interestingly, we did not found any conventional NLS by PSORT II analysis of survivin, despite reports on nuclear import of survivin after induction of apoptosis. ${ }^{21}$ Although we cannot exclude the existence of other as yet undefined NLS-functioning sequences, nuclear translocation of survivin may also be regulated by alternative mechanisms, including as yet unknown shuttle proteins, cytoplasmic retention factors or phosphorylation events. In this context, it is interesting to note that casein kinase II (CKII) increases the nuclear translocation of nucleoplasmin ${ }^{41}$ and that survivin, as well as survivin-2B, exhibit multiple sites for the action of CKII and other kinases. ${ }^{29}$ Moreover, targeted phosphorylation of survivin at $\mathrm{T}^{34}$ by the $\mathrm{p} 34^{\mathrm{cdc} 2}$-cyclinB1 complex was recently shown to be associated with nuclear translocation. ${ }^{15,23}$

In conclusion, our study provides data on differential subcellular localization of functionally divergent survivin variants. The cytoplasmic co-localization of anti-apoptotic survivin and non-antiapoptotic survivin-2B might permit an interactive fine-tuning of survivin actions. The accumulation of antiapoptotic survivin- $\Delta \mathrm{E} \times 3$ in the nuclear compartment might suggest a regulatory role for survivin- $\Delta \mathrm{Ex} 3$ during cell cycle progression. In contrast to the known association between survivin and centromeres or midbody microtubules during mitosis, no corresponding colocalization became evident for survivin- $\Delta \mathrm{Ex} 3$ or survivin-2B. These two survivin isoforms, therefore, may have functions and molecular interaction partners different from survivin in distinct phases of the cell cycle. Further studies with survivin variantspecific antibodies are clearly needed to elucidate the regulatory interactions of different survivin variants under physiological conditions. 


\section{Materials and Methods}

\section{Prediction of subcellular localization by PSORT II analysis}

We performed a PSORT II analysis, which is based on a pattern recognition algorithm called the k-nearest-neighbor method. ${ }^{42}$ The predictive accuracy of PSORT II had been assessed by 10 -fold crossvalidation using $1531 \mathrm{~S}$. cerevisiae sequences encoded in nuclear DNA. The sequences and localization sites used for prediction by PSORT II are derived from the SWISS-PROT and YPD databases. ${ }^{43,44}$ The PSORT II program displays the most probable sites for subcellular localization of proteins calculated on the basis of their amino acid sequence.

For detection of nuclear localization signals (NLS) by PSORT II, three different rules were used: 'pat4' recognizes a pattern composed of four basic amino acids ( $K$ or $\mathrm{R}$ ), or three basic amino acids ( $K$ or $\mathrm{R}$ ) with either $\mathrm{H}$ or $\mathrm{P}$; 'pat7' recognizes a pattern which starts with a $\mathrm{P}$ and is followed within the next three amino acids by a basic segment of four amino acids containing three K or R residues; a 'bipartite' NLS pattern which is composed of two basic amino acids, plus a ten amino acid spacer plus another basic region of five amino acids containing three basic amino acids. Moreover, a score which discriminates the localization tendency was calculated based on a neural network method. $^{45}$

The detection of nuclear exporting signals (NES) is not implemented in PSORT II, and, therefore, could not be analyzed.

\section{Cell lines and cultures}

HepG2 hepatoma cells and HeLa were maintained with RPMI medium or DMEM (Life Technologie/GibcoBRL, Karlsruhe, Germany), both supplemented with $10 \%$ heat-inactivated fetal calf serum (FCS), penicillin and streptomycin. Cells were cultivated at $37^{\circ} \mathrm{C}$ in an atmosphere with $5 \%(\mathrm{v} / \mathrm{v}) \mathrm{CO}_{2}$.

\section{Construction of expression vectors}

Reverse transcription (RT) reactions contained $2 \mu \mathrm{g}$ of total RNA, $1 \times \mathrm{RT}$ buffer (Promega, Heidelberg, Germany), $25 \mu \mathrm{M}$ of each dNTP, 10 pmol of sequence-specific RT-primer (5'-AGG AAC CTG CAG CTC AGA-3', corresponding to nucleotides 914-931 of the survivin antisense strand) or $0.06 \mu \mathrm{g}$ random primer (Stratagene, Heidelberg, Germany), $20 \mathrm{U}$ of RNAsin RNAse inhibitor (Promega), and $5 \mathrm{U}$ of AMV reverse transcriptase (Promega) in a final volume of $30 \mu \mathrm{l}$. The specific RT reactions were incubated at $50^{\circ} \mathrm{C}$ for $1 \mathrm{~h}$, random RT reactions at $37^{\circ} \mathrm{C}$ for $1 \mathrm{~h}$. PCR amplifications were performed on a PTC-100 Programmable Thermal Controller (Biozym Diagnostic, Hess. Oldendorf, Germany). Three $\mu$ l of cDNA mixture were subjected to amplification in a $50 \mu \mathrm{l}$ mixture containing $2.5 \mathrm{U}$ Taq polymerase, $1 \times$ PCR buffer (both from Qiagen), $25 \mu \mathrm{M}$ each of dATP, dCTP, dGTP, dTTP and $25 \mathrm{pmol}$ each of $5^{\prime}$ and $3^{\prime}$ primers. PCR conditions were: Initial denaturation for $2 \mathrm{~min}$ at $94^{\circ} \mathrm{C}$, followed by 30 cycles of denaturation at $94^{\circ} \mathrm{C}$ for $30 \mathrm{~s}$, annealing for $1 \mathrm{~min}$ at $62^{\circ} \mathrm{C}$, extension at $72^{\circ} \mathrm{C}$ for $1 \mathrm{~min}$, and a final extension at $72^{\circ} \mathrm{C}$ for $5 \mathrm{~min}$. To generate enhanced green fluorescent protein (GFP)- tagged survivin constructs, the coding sequences of the three survivin variants were PCRamplified as described above using the following primer sets: $5^{\prime}$-GTC GTC GGT ACC ATG GGT GCC CCG ACG TTG-3' (sense), and 5'CAG CAG GGA TCC ATC CAT GGC AGC CAG CTG CTC-3 (antisense) for survivin and survivin-2B; 5'-CAG CAG GGA TCC AGA CAT TGC TAA GGG GCC CAC A-3' (antisense) for survivin- $\triangle E \times 3$. The NLS-deleted survivin- $\triangle$ Ex3-GFP construct (lacking amino acids
$\mathrm{Arg}^{76}$ - Cys $^{92}$, designated as survivin- $\Delta$ Ex3- ${ }^{\text {NLS }}$-GFP) was generated as follows: both sequences flanking the NLS of survivin- $\Delta E \times 3$ were amplified separately by PCR with the following primer sets: $5^{\prime}$-GTC GTC GGT ACC ATG GGT GCC CCG ACG TTG-3' (sense); 5'-TTG CAT GGG GTC GTC AT-3'(antisense) and 5'-GCC GTG CCA TCG AGC-3' (sense); 5'-CAG CAG GGA TCC AGA CAT TGC TAA GGG GCC CAC (antisense). Afterwards the different PCR products were purified (Pharmacia Biotech Europe, Freiburg, Germany), ligated, and subsequently cloned in pEGFP-N3 (Clontech, Heidelberg, Germany) according to standard protocols.

All other PCR reactions were purified using Microspin S-300 columns too, digested with $K p n l$ and $B a m H I$, ligated into the mammalian expression vector pEGFP-N3, and cloned according to standard protocols. Bands of interest were eluted from agarose gels using the QIAquick gel extraction kit (Qiagen), ligated into the pGEMT-cloning vector (Promega) and cloned in accordance to standard protocols. Plasmid DNA containing the inserts was recovered employing the Plasmid Mini Kit (Qiagen), cycle sequenced and analyzed in an $\mathrm{ABI}$ Prism 310 sequencing apparatus (Applied Biosystems, Weiterstadt, Germany) using T7 or SP6 site specific primers.

\section{Transfection of HepG2 cells, induction of cell death, assessment of cell viability and inhibition of translation}

Two $\mu \mathrm{g}$ pEGFP-N3 vector control and survivin constructs were each co-transfected with $0.4 \mu \mathrm{g} \mathrm{pCMV} \beta$ by the DEAE-dextran protocol into HepG2 hepatoma cells according standard protocols. After culturing for $24 \mathrm{~h}$, cells were plated into 6-well plates and incubated with $250 \mathrm{ng} / \mathrm{ml}$ of the antibody $\mathrm{CH} 11$ (Immunotech, Marseille, France), that induces apoptosis via the CD95 pathway by crosslinking CD95 receptor molecules. ${ }^{46}$ Cell viability was measured after $24 \mathrm{~h}$ by scoring cells as alive or dead by trypan blue staining. Transfection experiments were performed at least three times as triplicates. To determine transfection efficiency, control cells were fixed $48 \mathrm{~h}$ after transfection ( $2 \%$ formaldehyde/0.2\% glutaraldehyde in PBS) and stained for $\beta$-galactosidase expression (0.1\% 5-bromo-4-chloro-3indolyl $\beta$-D-galactoside (X-Gal), $5 \mathrm{mM}$ potassium ferricyanide, $5 \mathrm{mM}$ potassium ferricyanide, $2 \mathrm{mM} \mathrm{MgCl} 2$ ). For determining the specific degradation of survivin and survivin- $\Delta \mathrm{Ex} 3$, cells were grown in six-well plates, transiently transfected using Polyfect reagent (Qiagen, Hilden, Germany) with the constructs containing survivin-EGFP, survivin$\triangle$ Ex3-EGFP and EGFP, respectively. Eighteen hours after transfection, cells were treated with $50 \mu \mathrm{g} / \mathrm{ml}$ cycloheximide (CHX) for 12, 24 and $48 \mathrm{~h}$. Afterwards, cells were harvested and proteins were extracted.

\section{Immunocytochemical analysis of subcellular localization}

Thirty-six hours after transfection, HepG2 cells were plated onto slides, fixed with methanol and labeled with Ki-67 antibody (Dianova, Hamburg, Germany). Ki-67 staining was detected with Cylll-coupled anti-mouse antibodies. Cell samples were analyzed using a Leica TCS-NT confocal laser scanning system (with an argon-krypton laser on a Leica DM IRB inverted microscope). Confocal microscopy images were acquired from two channels at $488 \mathrm{~nm}$ and $568 \mathrm{~nm}$ excitation wavelength. Emission was measured at $530 \pm 10 \mathrm{~nm}$ (green) and $>590 \mathrm{~nm}$ (red). Co-localization of the green (fluorescein) and red (CyIII) fluorescent dyes results in a yellow signal. Only samples which were prepared in parallel in all steps were compared, using the same adjustments for all parameters (i.e. laser power, filter settings, setting 
of the acousto-optical tuneable filter, pinhole, lens, voltages at the photo multiplier tubes, number of accumulated scans, format size and zoom, scan speed and z-step-size when whole thickness of the cell samples were analyzed).

For CENP-F staining, HeLa cells were transfected with constructs for survivin, survivin-2B or survivin- $\Delta E x 3$, using PolyFect (Qiagen, Hilden, Germany) according to the manufacturer's protocol. After incubation for $24 \mathrm{~h}\left(37^{\circ} \mathrm{C}, 5 \% \mathrm{CO}_{2}\right)$, HeLa cells were fixed with $2 \%$ para-formaldehyde for $10 \mathrm{~min}$ at room temperature. For centromere labeling, cells were incubated with rabbit polyclonal antibody CENP-F (Calbiochem, San Diego, CA, USA; dilution: 1:1000 in PBS, 5\% BSA and $0.5 \%$ Saponin). CENP-F binding was detected with Texas-Red coupled anti-rabbit-antibody (Zymed Lab. Inc., San Francisco, CA, USA; dilution: $1: 250$ in PBS, 5\% BSA and 0.5\% Saponin). Nuclei were counterstained with $0.1 \mu \mathrm{g} / \mathrm{ml}$ DAPI (Sigma-Aldrich, Taufkirchen, Germany) in PBS for $10 \mathrm{~min}$ at room temperature. Cell samples were analyzed using Zeiss Axioskop and Cytovision 3.52D software (Applied Imaging, Newcastle upon Tyne, UK).

\section{Immunoblotting}

Protein extracts of transfected HepG2 cells were electrophoresed on SDS-polyacrylamide gels and transferred to nitrocellulose membranes. The membrane was blocked overnight in blocking buffer (100 mM Tris/ $\mathrm{HCl}, \mathrm{pH} 7.5,150 \mathrm{mM} \mathrm{NaCl}, 0.2 \%$ Tween 20) plus 3\% non-fat dry milk/1\% BSA, incubated for $1 \mathrm{~h}$ at room temperature with polyclonal anti-human survivin antibody (Novus Biologicals, clone NB 500-201, Littleton, CO, USA), and incubated thereafter with the secondary antibody coupled with horseradish peroxidase for $1 \mathrm{~h}$ at room temperature. Detection was performed by incubation with the Lumi-Light substrate (Roche, Mannheim, Germany). Comparable loading was confirmed by $\alpha$-tubulin detection with an anti- $\alpha$-tubulin antibody (Sigma-Aldrich, Deisenhofen, Germany).

Blocking of translation by $\mathrm{CHX}: 10 \mu \mathrm{g}$ of protein extracts was subjected to immunoblotting. To detect the fusion proteins, we used a mouse monoclonal anti-GFP-antibody (JL8; Clontech, Heidelberg, Germany) diluted $1: 2000$, followed by incubation with a secondary, horseradish peroxidase-coupled antibody, diluted 1:2000.

\section{Note added in proof}

During the reviewing process of our manuscript, Rodriguez et al. (Exp. Cell. Res., 275:44, 2002) described corresponding observations on the subcellular localization of survivin- $\Delta \mathrm{Ex} 3$.

\section{Acknowledgements}

We thank Sigrid Khalil, Michael Ringler, and Martina Bellack for technical assistance. The results of this work are part of the Ph.D. thesis of $\mathrm{J}$ Liebmann and M Wenzel. This work was supported by a grant from the Deutsche Forschungsgemeinschaft (DFG).

\section{References}

1. Rudin CM and Thompson CB (1997) Apoptosis and disease: regulation and clinical relevance of programmed cell death. Annu. Rev. Med. 48: 267-281

2. Reed JC (1999) Dysregulation of apoptosis in cancer. J. Clin. Oncol. 17: 2941 2953
3. Aravind L, Dixit VM and Koonin EV (1999) The domains of death: evolution of the apoptosis machinery. Trends Biochem. Sci. 24: 47-53

4. Crook NE, Clem RJ and Miller LK (1993) An apoptosis-inhibiting baculovirus gene with a zinc finger-like motif. J. Virol. 67: 2168-2174

5. Uren AG, Coulson EJ and Vaux DL (1998) Conservation of baculovirus inhibitor of apoptosis repeat proteins (BIRPs) in viruses, nematodes, vertebrates and yeasts. Trends Biochem. Sci. 23: 159-162

6. Uren AG, Beilharz T, O'Connell MJ, Bugg SJ, van Driel R, Vaux DL and Lithgow T (1999) Role for yeast inhibitor of apoptosis (IAP)-like proteins in cell division. Proc. Natl. Acad. Sci. USA 96: 10170-10175

7. Wenzel M, Mahotka C, Krieg A, Bachmann A, Schmitt M, Gabbert HE and Gerharz CD (2000) Novel survivin-related members of the inhibitor of apoptosis (IAP) family. Cell Death. Differ. 7: 682-683

8. LaCasse EC, Baird S, Korneluk RG and MacKenzie AE (1998) The inhibitors of apoptosis (IAPs) and their emerging role in cancer. Oncogene 17: 3247-3259

9. Deveraux QL and Reed JC (1999) IAP family proteins-suppressors of apoptosis. Genes Dev. 13: 239-252

10. Ambrosini G, Adida C and Altieri DC (1997)A novel anti-apoptosis gene, survivin, expressed in cancer and lymphoma. Nat. Med. 3: 917-921

11. Tamm I, Wang Y, Sausville E, Scudiero DA, Vigna N, Oltersdorf T and Reed JC (1998) IAP-family protein survivin inhibits caspase activity and apoptosis induced by Fas (CD95), Bax, caspases, and anticancer drugs. Cancer Res. 58: $5315-5320$

12. Shin S, Sung BJ, Cho YS, Kim HJ, Ha NC, Hwang Jl, Chung CW, Jung YKand Oh $\mathrm{BH}$ (2001) An anti-apoptotic protein human survivin is a direct inhibitor of caspase-3 and -7. Biochemistry 40: 1117-1123

13. Verdecia MA, Huang H, Dutil E, Kaiser DA, Hunter T and Noel JP (2000) Structure of the human anti-apoptotic protein survivin reveals a dimeric arrangement. Nat. Struct. Biol. 7:602-608

14. Banks DP, Plescia J, Altieri DC, Chen J, Rosenberg SH, Zhang H and Ng SC (2000) Survivin does not inhibit caspase-3 activity. Blood 96: 4002-4003

15. O'Connor DS, Grossman D, Plescia J, LiF, Zhang H, Villa A, Tognin S, Marchisio PC and Altieri DC (2000) Regulation of apoptosis at cell division by p34cdc2 phosphorylation of survivin. Proc. Natl. Acad. Sci. USA 97: 13103-13107

16. Li F, Ambrosini G, Chu EY, Plescia J, Tognin S, Marchisio PC and Altieri DC (1998) Control of apoptosis and mitotic spindle checkpoint by survivin. Nature 396: $580-584$

17. Zhao J, Tenev T, Martins LM, Downward J and Lemoine NR (2000) The ubiquitinproteasome pathway regulates survivin degradation in a cell cycle-dependent manner. J. Cell Sci. 113: 4363-4371

18. Uren AG, Wong L, Pakusch M, Fowler KJ, Burrows FJ, Vaux DL and Choo KH (2000) Survivin and the inner centromere protein INCENP show similar cellcycle localization and gene knockout phenotype. Curr. Biol. 10: 1319-1328

19. Skoufias DA, MollinariC, Lacroix FB and Margolis RL (2000) Human survivin is a kinetochore-associated passenger protein. J. Cell Biol. 151: 1575-1582

20. Wheatley SP, Carvalho A, Vagnarelli P and Earnshaw WC (2001) INCENP is required for proper targeting of Survivin to the centromeres and the anaphase spindle during mitosis. Curr. Biol. 11: 886-890

21. Suzuki $A$, Hayashida M, Ito T, Kawano H, Nakano T, Miura M, Akahane $K$ and Shiraki K (2000) Survivin initiates cell cycle entry by the competitive interaction with Cdk4/p16(INK $\left.{ }^{4 a}\right)$ and Cdk2/cyclin E complex activation. Oncogene 19: 3225-3234

22. Suzuki A, Ito T, Kawano H, Hayashida M, Hayasaki Y, Tsutomi $Y$, Akahane $\mathrm{K}$, Nakano T, Miura M and Shiraki K (2000) Survivin initiates procaspase 3/p21 complex formation as a result of interaction with $\mathrm{Cdk} 4$ to resist Fas-mediated cell death. Oncogene 19: 1346-1353

23. Fortugno P, Wall NR, Giodini A, O'Connor DS, Plescia J, Padgett KM, Tognin S, Marchisio PC and Altieri DC (2002) Survivin exists in immunochemically distinct subcellular pools and is involved in spindle microtubule function. J. Cell Sci. 115: $575-585$

24. Jiang X, Wilford C, Duensing S, Munger K, Jones G and Jones D (2001) Participation of Survivin in mitotic and apoptotic activities of normal and tumorderived cells. J. Cell Biochem. 83: 342-354

25. Mesri M, Morales-Ruiz M, Ackermann EJ, Bennett CF, Pober JS, Sessa WC and Altieri DC (2001) Suppression of vascular endothelial growth factor-mediated endothelial cell protection by survivin targeting. Am. J. Pathol. 158: 1757-1765 
26. Kornacker M, Verneris MR, Kornacker B, Scheffold C and Negrin RS (2001) Survivin expression correlates with apoptosis resistance after lymphocyte activation and is found preferentially in memory T cells. Immunol. Lett. 76: 169173

27. Adida C, Berrebi D, Peuchmaur M, Reyes-Mugica M and Altieri DC (1998) Antiapoptosis gene, survivin, and prognosis of neuroblastoma. Lancet 351: $882-$ 883

28. Kawasaki H, Altieri DC, Lu CD, Toyoda M, Tenjo T and Tanigawa N (1998) Inhibition of apoptosis by survivin predicts shorter survival rates in colorectal cancer. Cancer Res. 58: 5071-5074

29. Mahotka C, Wenzel M, Springer E, Gabbert HE and Gerharz CD (1999) SurvivindeltaEx3 and survivin-2B: two novel splice variants of the apoptosis inhibitor survivin with different antiapoptotic properties. Cancer Res. 59: 6097-6102

30. Islam A, Kageyama H, Hashizume K, Kaneko Y and Nakagawara A (2000) Role of survivin, whose gene is mapped to $17 q 25$, in human neuroblastoma and identification of a novel dominant-negative isoform, survivin- beta/2B. Med. Pediatr. Oncol. 35: 550-553

31. Scholzen T and Gerdes J (2000) The Ki-67 protein: from the known and the unknown. J. Cell Physiol. 182: 311-322

32. Liao H, Winkfein RJ, Mack G, Rattner JB and Yen TJ (1995) CENP-F is a protein of the nuclear matrix that assembles onto kinetochores at late $\mathrm{G} 2$ and is rapidly degraded after mitosis. J. Cell Biol. 130: 507-518

33. Jans DA and Hübner S (1996) Regulation of protein transport to the nucleus - the central role of phosphorylation. Physiol. Rev. 76: 651-685

34. Otterlei M, Haug T, Nagelhus TA, Slupphaug G, Lindmo T and Krokan HE (1998) Nuclear and mitochondrial splice forms of human uracil-DNA glycosylase contain a complex nuclear localisation signal and a strong classica mitochondrial localisation signal, respectively. Nucleic Acids Res. 26: 4611 4617

35. Conway EM, Pollefeyt S, Cornelissen J, DeBaere I, Steiner-Mosonyi M, Ong K Baens M, Collen D and Schuh AC (2000) Three differentially expressed survivin cDNA variants encode proteins with distinct antiapoptotic functions. Blood 95: $1435-1442$
36. Muchmore SW, Chen J, Jakob C, Zakula D, Matayoshi ED, Wu W, Zhang H, Li F, $\mathrm{Ng}$ SC and Altieri DC (2000) Crystal structure and mutagenic analysis of the inhibitor-of-apoptosis protein survivin. Mol. Cell 6: 173-182

37. Chantalat L, Skoufias DA, Kleman JP, Jung B, Dideberg $O$ and Margolis RL (2000) Crystal structure of human survivin reveals a bow tie-shaped dimer with two unusual alpha-helical extensions. Mol. Cell 6: 183-189

38. Li F and Altieri DC (1999) Transcriptional analysis of human survivin gene expression. Biochem. J. 344 Pt 2: 305-311

39. Krieg A, Mahotka C, Krieg T, Grabsch H, Muller W, Takeno S, Suschek CV, Heydthausen M, Gabbert HE and Gerharz CD (2002) Expression of different survivin variants in gastric carcinomas: first clues to a role of survivin-2B in tumour progression. Br. J. Cancer 86: 737-743

40. Mahotka C, Krieg T, Krieg A, Wenzel M, Suschek CV, Heydthausen M, Gabbert $\mathrm{HE}$ and Gerharz CD (2002) Distinct in vivo expression patterns of survivin splice variants in renal cell carcinomas. Int. J. Cancer 100 (1): 30-36

41. Robbins J, Dilworth SM, Laskey RA and Dingwall C (1991) Two interdependent basic domains in nucleoplasmin nuclear targeting sequence: identification of a class of bipartite nuclear targeting sequence. Cell 64: 615-623

42. Nakai K and Horton P (1999) PSORT: a program for detecting sorting signals in proteins and predicting their subcellular localization. Trends Biochem. Sci. 24: $34-36$

43. Bairoch A and Apweiler R (1997) The SWISS-PROT protein sequence data bank and its supplement TrEMBL. Nucleic Acids Res. 25: 31-36

44. Garrels JI (1996) YPD-A database for the proteins of Saccharomyces cerevisiae. Nucleic Acids Res. 24: 46-49

45. Reinhardt A and Hubbard T (1998) Using neural networks for prediction of the subcellular location of proteins. Nucleic Acids Res. 26: 2230-2236

46. Garrone P, Neidhardt EM, Garcia E, Galibert L, van Kooten C and Banchereau J (1995) Fas ligation induces apoptosis of CD40-activated human B lymphocytes. J. Exp. Med. 182: 1265-1273 